\title{
A Comparative Study of the Behavioral Profile of the Behavioral Variant of Frontotemporal Dementia and Parkinson's Disease Dementia
}

\author{
Dinesh Saini Adreesh Mukherjee Arijit Roy Atanu Biswas \\ Department of Neurology, Bangur Institute of Neurosciences, and Institute of \\ Post Graduate Medical Education and Research, Kolkata, India
}

\section{Keywords}

Behavioral variant of frontotemporal dementia - Behavioral symptoms - Cortical dementia .

Parkinson's disease dementia $\cdot$ Subcortical dementia

\begin{abstract}
Background: Executive dysfunction is the common thread between pure cortical dementia like the behavioral variant of frontotemporal dementia (bvFTD) and subcortical dementia like Parkinson's disease dementia (PDD). Although there are clinical and cognitive features to differentiate cortical and subcortical dementia, the behavioral symptoms differentiating these 2 conditions are still not well known. Objective: To evaluate the behavioral profile of bvFTD and PDD and compare them to find out which behavioral symptoms can differentiate between the two. Methods: Twenty consecutive patients with bvFTD ( $>1$ year after diagnosis) and 20 PDD patients were recruited according to standard diagnostic criteria. Behavioral symptoms were collected from the reliable caregiver by means of a set of questionnaires and then compared between the 2 groups. Results: bvFTD patients had more severe disease and more behavioral symptoms than PDD. bvFTD patients were different from PDD patients due to their significantly greater: loss of basic emotion ( $p<0.001$, odds ratio [OR] 44.33), loss of awareness of pain $(p<0.001$, OR 44.33), disinhibition ( $p<0.001$, OR 35.29), utilization phenomenon $(p=0.008$, OR 22.78), loss of taste discrimination $(p<0.001$, OR 17), neglect of hygiene $(p=$ 0.001 , OR 13.22), loss of embarrassment ( $p=0.003$, OR 10.52), wandering ( $p=0.004$, OR 9.33), pacing $(p=0.014$, OR 9), selfishness $(p=0.014$, OR 9), increased smoking $(p=0.014$, OR 9$)$, increased alcohol consumption ( $p=0.031$, OR 7.36), social avoidance $(p=0.012$, OR 6.93), mutism ( $p=0.041$, OR 5.67), and failure to recognize objects ( $p=0.027$, OR 4.33). The bvFTD patients were also significantly less suspicious ( $p=0.001$, OR 0.0295$)$, less inclined to have a false belief that people were in their home ( $p=0.014$, OR 0.11) and had fewer visual illusions/ hallucinations ( $p=0.004$, OR 0.107) than PDD patients. Conclusion: Behavioral symptoms are helpful to distinguish bvFTD from PDD, and thus also cortical dementia with frontal-lobe dysfunction from subcortical dementia.




\section{Introduction}

The frontal lobe plays a crucial role in human behavior. Some of the most striking neurobehavioral syndromes are coupled with frontal-lobe dysfunction. Cummings [1] described 3 clinical syndromes that involve frontal-lobe circuitry: (a) apathy and akinesia resulting from damage to the mesial frontal or anterior cingulate pathway, (b) disinhibition, emotional dysregulation, and distractibility resulting from damage to the orbitofrontal cortex, and (c) deficits in executive function and motor programming due to damage to the dorsolateral prefrontal cortex. Among degenerative neurological disorders, the behavioral variant of frontotemporal dementia (bvFTD) presents with behavioral alterations very early in the disease course, even before cognitive dysfunction becomes evident. As the mesial frontal lobe is connected to the hippocampus and the orbital frontal lobe is connected to the inferior and superior temporal lobes, cortical dementias like Alzheimer's disease (AD) also demonstrate marked behavioral changes [2]. The prefrontal cortex also has extensive connections with subcortical structures such as the basal ganglia and thalamus, and disturbance of these frontosubcortical circuits also results in abnormal behaviors [1,3]. Thus, behavioral abnormalities occur both in cortical dementia (bvFTD, AD, etc.) and subcortical dementia (Huntington's disease, Parkinson's disease dementia [PDD], and vascular dementia [VaD]) [4]. Common behavioral abnormalities observed in PDD include depression, anxiety, and apathy. PDD patients may also have excessive daytime sleepiness, visual hallucinations, delusions, paranoia, and confusion [5]. The characteristics of bvFTD are early and profound alterations in personality, social conduct, and behavior. However, these symptoms are not specific to this disease. There is a significant overlap of the symptoms of bvFTD and psychiatric diseases such as bipolar disorder and schizophrenia [6]. Degeneration of the dorsolateral prefrontal cortex also leads to disturbed executive function in patients with bvFTD.

The cognitive disturbance of subcortical dementia manifests as impairment of executive function, impaired attention, reduced speed of information processing, and retrieval defect in memory tasks that improve with cueing. Patients of subcortical dementia often exhibit frontal behavioral features like that of bvFTD, which can create diagnostic difficulties [7]. Clinicians rely upon the cognitive profile and presence of additional motor features and sphincter disturbance to distinguish subcortical from cortical dementia. To clinically differentiate between various dementias, efforts have been made to investigate the behavioral and psychiatric symptoms of these patients [8-10]. However, only a few studies have attempted to explore the behavioral profile to differentiate cortical and subcortical dementia [2]. We hypothesized that core frontal-lobe behaviors, such as impaired social cognition, disinhibition, alteration of feeding, sexual behavior and sensory perceptions, environmental dependency, etc. are relatively uncommon in subcortical dementia, and can thus be used to clinically differentiate cortical and subcortical dementia. We used a questionnaire prepared by Bathgate et al. [11] to capture the behavior of patients. This questionnaire was developed with the primary aim of determining the discriminating value of behavioral characteristics in differentiating bvFTD from 2 common forms of dementia, $\mathrm{AD}$, and subcortical vascular dementia. To differentiate FTD from AD, informant-based behavioral interviews were also developed by Bozeat et al. [12], and Ikeda et al. [13]. However, Bathgate et al. [11] demonstrated that changes in emotions and insight, selfishness, disinhibition, personal neglect, gluttony and sweet food preference, wandering, stereotypies, loss of sensitivity to pain, echolalia, and mutism were more characteristic of bvFTD, and differentiated most bvFTD from AD and vascular dementia. The Frontal Systems Behavior Scale (FrSBe) is another questionnaire which measures behavior associated with frontal subcortical deficits, but it tests only 3 items, apathy, disinhibition, and executive dysfunction [2].

\section{Karger'}


We planned to evaluate the behavioral profiles of bvFTD as representative of cortical dementia, and PDD as representative of subcortical dementia, and compare them to find out which behavioral symptoms differentiate cortical and subcortical dementia.

\section{Methods}

This was a questionnaire-based, observational, comparative study conducted between March 2015 and October 2016. A purposive sampling technique was employed for the recruitment of patients from the Cognitive and Movement Disorders Clinic (MDC) of our institute. We selected patients with probable bvFTD, diagnosed according to the international consensus criteria for bvFTD [6]. Patients with bvFTD of $>1$ year's duration and regularly attending follow-up were included in the study. Parkinsonian patients who had regularly attended follow-up at the MDC for $>5$ years and had received a primary diagnosis of idiopathic Parkinson's disease (IPD) were recruited. At the point of their recruitment, these patients had cognitive dysfunction and fulfilled the clinical diagnostic criteria for PDD [14].

We excluded (a) patients and caregivers who were not willing to participate in the study, (b) patients with overlapping features of both degenerative and vascular dementia, (c) patients with such severe dementia that they could not be assessed, and (d) patients with incomplete clinical evaluation and brain imaging data.

A detailed history was obtained from each patient/a reliable caregiver, and neurological examinations and cognitive functions assessment were conducted. Their attention was tested using the continuous performance task/test, digit span test, and serial subtraction test. Memory was tested with the verbal learning test as per the Kolkata Cognitive Screening Battery [15]. For language, we used the Bengali version of the Western Aphasia Battery [16]. Visuospatial domain was tested using the letter cancellation task and line bisection test, while visuoperceptual testing was done via dot-counting, fragmented letters, and progressive silhouettes. Frontal-lobe function testing was done using the Frontal Assessment Battery (FAB) [17]. All the above tools and tests were validated for the Bengali language, and we have used them in previous studies as well $[18,19]$.

All patient underwent detailed lab investigations, including complete blood count, erythrocyte sedimentation rate, blood biochemical tests like the thyroid function test, levels of serum Vitamin $\mathrm{B}_{12}$, fasting and postprandial blood glucose, and glycosylated hemoglobin. Lipid profile was obtained, and tests of liver and renal function as well as magnetic resonance imaging (MRI) of the brain were conducted.

Behavioral symptoms were collected from a reliable caregiver via a set of questionnaires developed by Bathgate et al. [11]. In this checklist, various behaviors were subcategorized into 7 major categories - affect and social, sensory behaviors, eating and vegetative behaviors, repetitive and-compulsive ritual behaviors, environmental dependency, cognitively mediated behaviors, and behaviors related to psychosis. We used the Bengali translated version. It was emphasized that a "symptom" should reveal a remarkable change from the patient's premorbid condition and not a longstanding trait. We ensured that each symptom being assessed was understood by the caregivers. The response to each question was recorded as the absence or presence of symptoms. Interviews were conducted by a single interviewer.

\section{Statistical Analysis}

For statistical analysis, data were entered in a Microsoft Excel spreadsheet and then analyzed by SPSS v24.0 (SPSS Inc., Chicago, IL, USA) and GraphPad Prism v5. We used descriptive statistics for analyzing the baseline demographics, Bengali Mental Status Examination (BMSE), Clinical Dementia Rating (CDR), and FAB scores. Data were summarized as 
Dementia and Geriatric Cognitive Disorders Extra

Table 1. Demographic and cognitive characteristics of bvFTD and PDD
Table 2. Frequency of behavioral domains affected in bvFTD and PDD

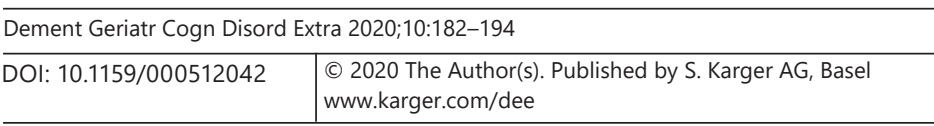

\begin{tabular}{lccl}
\hline & bvFTD & PDD & $p$ value \\
\hline$N$ & 20 & 20 & - \\
Age, years & $56.60 \pm 10.26$ & $62.25 \pm 7.26$ & 0.052 \\
Gender $\quad$ & & \\
$\quad$ Male, $n$ & 15 & 13 & 0.49 \\
$\quad$ Female, $n$ & 5 & 7 & \\
Duration of illness, years & $3.85 \pm 1.57$ & $4.3 \pm 2.25$ & 0.718 \\
Years of education & $6.35 \pm 5.16$ & $7.35 \pm 4.93$ & 0.535 \\
BMSE score & $9.05 \pm 5.37$ & $20.60 \pm 5.63$ & $0.0001^{*}$ \\
FAB score & $1.95 \pm 2.43$ & $8.25 \pm 6.02$ & $0.0001^{*}$ \\
CDR & $1.29 \pm 0.37$ & $0.89 \pm 0.35$ & $0.0001^{*}$ \\
\hline
\end{tabular}

Values express mean \pm SD, unless otherwise indicated. Asterisks denote significance. bvFTD, behavioral variant of frontotemporal dementia; PDD, Parkinson's disease dementia; BMSE, Bengali Mental Status Examination; FAB, Frontal Assessment Battery; CDR, Clinical Dementia Rating.

\begin{tabular}{llll}
\hline Type of dementia & \multicolumn{2}{l}{$\begin{array}{l}\text { Number of behavioral } \\
\text { domains affected }\end{array}$} & $p$ value \\
\cline { 2 - 3 }$<3$ & $\geq 3$ & \\
\hline bvFTD, $n(\%)$ & 0 & $20(100)$ & 0.047 \\
PDD, $n(\%)$ & $5(25)$ & $15(75)$ & \\
\hline
\end{tabular}

bvFTD, behavioral variant of frontotemporal dementia; PDD, Parkinson's disease dementia.

mean and SD for continuous variables and $n(\%)$ for categorical variables. The various domains of behavioral symptoms in the bvFTD and PDD patients were compared by $\chi^{2} /$ Fisher's exact test. For the comparison of continuous variables between groups, the Mann-Whitney U test was performed. A $p$ value $<0.05$ was considered significant.

\section{Results}

Twenty bvFTD patients and 20 PDD were recruited for the study. Demographic profiles of our patients are given in Table 1. The bvFTD patients were younger than the PDD patients, but the difference was not statistically significant. There was male predominance in both groups. The mean duration of dementia at presentation was $3.85( \pm 1.57)$ years in the bvFTD group, i.e., comparable to the duration of dementia in the PDD group ( $4.3 \pm 2.25$ years). There was also no significant difference in years of education between bvFTD $(6.35 \pm 5.16)$ and PDD (7.35 \pm 4.93$)$ patients. bvFTD patients were cognitively more impaired than PDD patients.

bvFTD patients had more affected behavioral domains than PDD patients. While all $(100 \%)$ bvFTD patients had $\geq 3$ domains of behavioral symptoms, only $25 \%$ of PDD patients had $<3$ affected domains ( $p=0.047$ ). Table 2 shows the distribution of domains affected in the 2 groups of patients. Overall, all major behavioral domains were more commonly observed in bvFTD than in PDD, except for neuropsychiatric behavior (Table 3). 
Table 3. Comparison of behavioral symptoms in bvFTD and PDD

\begin{tabular}{|c|c|c|c|c|}
\hline & bvFTD, $n(\%)$ & PDD, $n(\%)$ & $p$ value & OR $(95 \% \mathrm{CI})$ \\
\hline \multicolumn{5}{|l|}{ A. Affect and social behavior } \\
\hline Loss of basic emotion & $19(95.0)$ & $6(30.0)$ & $<0.001^{*}$ & $44.33(4.78-410.94)$ \\
\hline Exaggerated emotional display & $5(25.0)$ & $3(15.0)$ & 0.694 & \\
\hline Loss of embarrassment & $13(65.0)$ & $3(15.0)$ & $0.003^{*}$ & $10.52(2.27-48.76)$ \\
\hline Irritability & $14(70.0)$ & $11(55.0)$ & 0.327 & \\
\hline Aggression & $11(55.0)$ & $5(25.0)$ & 0.053 & \\
\hline Loss of insight & $19(95.0)$ & $16(80.0)$ & 0.342 & \\
\hline Excessive worrying & $12(60.0)$ & $8(40.0)$ & 0.206 & \\
\hline Selfishness & $10(50.0)$ & $2(10.0)$ & $0.014^{*}$ & $9.00(1.64-49.45)$ \\
\hline Disinhibition & $19(95.0)$ & $7(35.0)$ & $<0.001^{*}$ & $35.29(3.87-321.93)$ \\
\hline Social avoidance & $17(85.0)$ & $9(45.0)$ & $0.012^{*}$ & $6.93(1.53-31.38)$ \\
\hline Seeks out social contact & $4(20.0)$ & $8(40.0)$ & 0.301 & \\
\hline Neglect of hygiene & $17(85.0)$ & $6(30.0)$ & $0.001^{*}$ & $13.22(2.79-62.67)$ \\
\hline Loss of interest & $18(90.0)$ & $13(65.0)$ & 0.127 & \\
\hline \multicolumn{5}{|l|}{ B. Sensory behavior } \\
\hline Loss of awareness of pain & $14(70.0)$ & $1(5.0)$ & $<0.001^{*}$ & $44.33(4.78-410.94)$ \\
\hline Loss of smell & $11(55.0)$ & $12(60.0)$ & 0.749 & \\
\hline Exaggerated sensory response & $6(30.0)$ & $4(20.0)$ & 0.716 & \\
\hline Exaggerated heat/cold response & $7(35.0)$ & $5(25.0)$ & 0.490 & \\
\hline \multicolumn{5}{|l|}{ C. Eating and vegetative behaviors } \\
\hline Preference for sweet foods & $6(30.0)$ & $4(20.0)$ & 0.716 & \\
\hline Preference for savory foods & $1(5.0)$ & $1(5.0)$ & 1.000 & \\
\hline Food fads & $2(10.0)$ & $3(15.0)$ & 1.000 & \\
\hline Loss of discrimination & $17(85.0)$ & $5(25.0)$ & $<0.001^{*}$ & $17.00(3.46-83.44)$ \\
\hline Overeats & $8(40.0)$ & $2(10.0)$ & 0.065 & \\
\hline Eats continually if food is present & $6(30.0)$ & $2(10.0)$ & 0.235 & \\
\hline Steals food from others' plates & $5(25.0)$ & $2(10.0)$ & 0.407 & \\
\hline Seeks out food & $6(30.0)$ & $2(10.0)$ & 0.235 & \\
\hline Crams food & $5(25.0)$ & $2(10.0)$ & 0.407 & \\
\hline Increased alcohol consumption & $9(45.0)$ & $2(10.0)$ & $0.031^{*}$ & $7.36(1.34-40.55)$ \\
\hline Increased smoking & $10(50.0)$ & $2(10.0)$ & $0.014^{*}$ & $9.00(1.64-49.45)$ \\
\hline Oral exploration of objects & $5(25.0)$ & $1(5.0)$ & 0.182 & \\
\hline Wandering & $14(70.0)$ & $4(20.0)$ & $0.004^{*}$ & $9.33(2.18-39.96)$ \\
\hline Pacing & $10(50.0)$ & $2(10.0)$ & $0.014^{*}$ & $9.00(1.64-49.45)$ \\
\hline Hyposexuality & $10(50.0)$ & $6(30.0)$ & 0.197 & \\
\hline Hypersexuality & $4(20.0)$ & $2(10.0)$ & 0.661 & \\
\hline Hypersomnia & $6(30.0)$ & $5(25.0)$ & 0.723 & \\
\hline Hyposomnia & $10(50.0)$ & $10(50.0)$ & 1.000 & \\
\hline \multicolumn{5}{|l|}{ D. Repetitive and compulsive behaviors } \\
\hline Simple motor stereotypies & $7(35.0)$ & $2(10.0)$ & 0.127 & \\
\hline Complex motor routines & $3(15.0)$ & 0 & 0.231 & \\
\hline Paces fixed route & $4(20.0)$ & $1(5.0)$ & 0.342 & \\
\hline Verbal stereotypies/perseverations & $5(25.0)$ & $3(15.0)$ & 0.695 & \\
\hline Repetitive themes & $1(5.0)$ & 0 & 1.000 & \\
\hline Counts objects & $1(5.0)$ & $2(10.0)$ & 1.000 & \\
\hline Aligns objects & $2(10.0)$ & 0 & 0.487 & \\
\hline Adherence to daily routine & $3(15.0)$ & $1(5.0)$ & 0.605 & \\
\hline Overconcern with cleanliness & 0 & $1(5.0)$ & 1.000 & \\
\hline Clock-watching & $4(20.0)$ & $5(25.0)$ & 1.000 & \\
\hline Excessive checking & $2(10.0)$ & $1(5.0)$ & 1.000 & \\
\hline Excessive attention to detail & $2(10.0)$ & $3(15.0)$ & 1.000 & \\
\hline Upset if routine disrupted & $3(15.0)$ & $3(15.0)$ & 1.000 & \\
\hline Needs to do things immediately & $2(10.0)$ & $2(10.0)$ & 1.000 & \\
\hline Unusual toileting routines & $8(40.0)$ & $9(45.0)$ & 0.749 & \\
\hline Superstitious rituals & 0 & $1(5.0)$ & 1.000 & \\
\hline
\end{tabular}




\section{Disorders Extra}

Table 3 (continued)

\begin{tabular}{|c|c|c|c|c|}
\hline & bvFTD, $n(\%)$ & PDD, $n(\%)$ & $p$ value & OR $(95 \% \mathrm{CI})$ \\
\hline \multicolumn{5}{|l|}{ E. Environmental dependency-related behaviors } \\
\hline Hoarding & $2(10.0)$ & $2(10.0)$ & 1.000 & \\
\hline Touches or handles objects & $6(30.0)$ & $2(10.0)$ & 0.235 & \\
\hline Echolalia & $4(20.0)$ & 0 & 0.106 & \\
\hline Echopraxia & $2(10.0)$ & 0 & 0.487 & \\
\hline Utilization phenomena & $7(35.0)$ & 0 & $0.008^{*}$ & $22.78(1.2-432.61)$ \\
\hline Reads aloud notices & $2(10.0)$ & $2(10.0)$ & 1.000 & \\
\hline \multicolumn{5}{|l|}{ F. Cognitively mediated behaviors } \\
\hline Mislays objects & $13(65.0)$ & $8(40.0)$ & 0.113 & \\
\hline Lost in familiar surroundings & $9(45.0)$ & $7(35.0)$ & 0.519 & \\
\hline Disorientation in own home & $11(55.0)$ & $8(40.0)$ & 0.342 & \\
\hline Fails to recognize objects & $13(65.0)$ & $6(30.0)$ & $0.027^{*}$ & $4.33(1.15-16.32)$ \\
\hline Difficulty locating objects & $12(60.0)$ & $10(50.0)$ & 0.525 & \\
\hline Uses wrong words & $9(45.0)$ & $5(25.0)$ & 0.185 & \\
\hline Mutism & $10(50.0)$ & $3(15.0)$ & $0.041^{*}$ & $5.67(1.25-25.61)$ \\
\hline \multicolumn{5}{|l|}{$\begin{array}{l}\text { G. Psychosis-related behaviors } \\
\text { (hallucinations and delusions) }\end{array}$} \\
\hline Suspiciousness & 0 & $9(45.0)$ & $0.001^{*}$ & $0.0295(0.002-0.56)$ \\
\hline Delusions of theft & $4(20.0)$ & $9(45.0)$ & 0.176 & \\
\hline False belief that people are in their home & $2(10.0)$ & $10(50.0)$ & $0.014^{*}$ & $0.11(0.02-0.61)$ \\
\hline Misidentification phenomena & $5(25.0)$ & $11(55.0)$ & 0.053 & \\
\hline Visual illusions/hallucinations & $4(20.0)$ & $14(70.0)$ & $0.004^{*}$ & $0.107(0.025-0.46)$ \\
\hline Auditory illusions/hallucinations & $2(10.0)$ & $3(15.0)$ & 1.000 & \\
\hline
\end{tabular}

Asterisks denote significance. OR, odds ratio; CI, confidence interval; bvFTD, behavioral variant of frontotemporal dementia; PDD, Parkinson's disease dementia.

Abnormal affect and social behavior were observed in all bvFTD patients and in $80 \%$ of the PDD patients. Regarding social behavior, the following subdomains were significantly more common in bvFTD than in PDD: loss of basic emotion ( $p<0.001$, odds ratio [OR] 44.33), disinhibition ( $p<0.001$, OR 35.29), neglect of hygiene ( $p=0.001$, OR 13.22), loss of embarrassment ( $p=0.003$, OR 10.52), social avoidance ( $p=0.012$, OR 6.93), and selfishness ( $p=$ 0.014 , OR 9). Although most other subdomains were also observed more in bvFTD than in PDD, the differences were not statistically significant.

Abnormal sensory behavior was also more common in bvFTD than PDD. Loss of the sensation of pain was significantly more common in bvFTD ( $p<0.001$, OR 44.33). Loss of smell as well as exaggerated heat and cold responses were observed to be almost similar in the 2 groups.

Eating and vegetative behavior were significantly more abnormal in patients with bvFTD (85\%) than in those with PDD (45\%). The loss of taste discrimination $(p<0.001$, OR 17), increased consumption of alcohol ( $p=0.031$, OR 7.36), and increased smoking ( $p=0.014$, OR 9 ) were significantly more evident in bvFTD. Although not statistically significant, overeating was reported more frequently in bvFTD $(p=0.065)$. Of the vegetative behaviors, wandering ( $p=0.004$, OR 9.33) and pacing ( $p=0.014$, OR 9) were found to be significantly more common in bvFTD, while other behaviors were common but did not attain statistical significance.

In the repetitive and compulsive behavior domain, no subdomain was significantly more common in either group. However, simple and complex motor stereotypies, and verbal stereotypies or perseverations were reported more commonly in bvFTD. 
Table 4. Cognitive performance in relation to behavioral symptoms in PDD

\begin{tabular}{|c|c|c|c|c|}
\hline \multirow[t]{3}{*}{ Behavioral symptoms } & \multicolumn{4}{|l|}{ PDD } \\
\hline & \multicolumn{2}{|l|}{ BMSE } & \multicolumn{2}{|l|}{ FAB } \\
\hline & mean \pm SD & $p$ value & mean \pm SD & $p$ value \\
\hline \multicolumn{5}{|l|}{ Loss of basic emotion } \\
\hline Yes & $14.33 \pm 4.457$ & $0.002^{*}$ & $5.17 \pm 3.601$ & 0.179 \\
\hline No & $23.29 \pm 3.583$ & & $9.57 \pm 6.465$ & \\
\hline \multicolumn{5}{|l|}{ Loss of embarrassment } \\
\hline Yes & $14.33 \pm 4.041$ & 0.093 & $4.00 \pm 3.464$ & 0.179 \\
\hline No & $21.71 \pm 5.193$ & & $9.00 \pm 6.134$ & \\
\hline \multicolumn{5}{|l|}{ Social avoidance } \\
\hline Yes & $19.44 \pm 6.023$ & 0.331 & $6.78 \pm 6.476$ & 0.23 \\
\hline No & $21.55 \pm 5.392$ & & $9.45 \pm 5.646$ & \\
\hline \multicolumn{5}{|l|}{ Neglect of hygiene } \\
\hline Yes & $16.17 \pm 7.574$ & 0.076 & $7.67 \pm 7.312$ & 0.718 \\
\hline No & $22.50 \pm 3.368$ & & $8.50 \pm 5.681$ & \\
\hline \multicolumn{5}{|l|}{ Loss of interest } \\
\hline Yes & $19.00 \pm 6.055$ & 0.081 & $7.77 \pm 6.457$ & 0.438 \\
\hline No & $23.57 \pm 3.359$ & & $9.14 \pm 5.49$ & \\
\hline \multicolumn{5}{|l|}{ Disinhibition } \\
\hline Yes & $19.00 \pm 6.191$ & 0.438 & $4.57 \pm 4.429$ & $0.019 *$ \\
\hline No & $21.46 \pm 5.364$ & & $10.23 \pm 5.96$ & \\
\hline \multicolumn{5}{|c|}{ Verbal stereotypies/perseverations } \\
\hline Yes & $26.00 \pm 2.646$ & 0.072 & $17.33 \pm 1.155$ & $0.007 *$ \\
\hline No & $19.65 \pm 5.511$ & & $6.65 \pm 4.974$ & \\
\hline \multicolumn{5}{|c|}{ Needs to do things immediately } \\
\hline Yes & $28.50 \pm 0.707$ & $0.011^{*}$ & $14.00 \pm 2.828$ & 0.211 \\
\hline No & $19.72 \pm 5.222$ & & $7.61 \pm 5.982$ & \\
\hline \multicolumn{5}{|c|}{ Exaggerated emotional display } \\
\hline Yes & $24.00 \pm 5.0$ & 0.358 & $12.00 \pm 8.718$ & 0.416 \\
\hline No & $20.00 \pm 5.657$ & & $7.59 \pm 5.523$ & \\
\hline \multicolumn{5}{|l|}{ Irritability } \\
\hline Yes & $20.91 \pm 5.449$ & 0.71 & $9.18 \pm 6.493$ & 0.766 \\
\hline No & $20.22 \pm 6.160$ & & $7.11 \pm 5.555$ & \\
\hline \multicolumn{5}{|l|}{ Aggression } \\
\hline Yes & $22.00 \pm 4.123$ & 0.735 & $11.40 \pm 6.229$ & 0.306 \\
\hline No & $20.13 \pm 6.105$ & & $7.20 \pm 5.784$ & \\
\hline \multicolumn{5}{|l|}{ Excessive worrying } \\
\hline Yes & $23.63 \pm 3.021$ & 0.082 & $11.88 \pm 5.222$ & $0.02^{*}$ \\
\hline No & $18.58 \pm 6.156$ & & $5.83 \pm 5.424$ & \\
\hline \multicolumn{5}{|l|}{ Seeks out social contact } \\
\hline Yes & $22.63 \pm 2.56$ & 0.343 & $9.13 \pm 5.139$ & 0.384 \\
\hline No & $19.25 \pm 6.757$ & & $7.67 \pm 6.706$ & \\
\hline
\end{tabular}

Asterisks denote significance. PDD, Parkinson's disease dementia; BMSE, Bengali Mental Status Examination; FAB, Frontal Assessment Battery.

While $40 \%$ of bvFTD patients had environmental dependency, only $15 \%$ of PDD patients showed similar behavior. Utilization behavior was observed in 35\% of the bvFTD but there was none in the PDD group ( $p=0.008$, OR 22.78). Although the differences did not attain significance, echolalia (20\%) and echopraxia (10\%) were observed only in bvFTD patients.

Cognitively mediated behavior was also more common in bvFTD (70\%) than in PDD (60\%). Failure to recognize objects ( $p=0.027$, OR 4.33) and mutism ( $p=0.041$, OR 5.67) were significantly more common in bvFTD. 
Of all these behavioral symptoms, neuropsychiatric behavior was more commonly observed in PDD (80\%) than in bvFTD (50\%). Suspiciousness ( $p=0.001,0 \mathrm{R} 0.0295)$, the false belief that people were in their home ( $p=0.014$, OR 0.11$)$ and visual illusions/hallucinations ( $p=0.004$, OR 0.107) were significantly less common in bvFTD than in PDD. Although not statistically significant, the misidentification phenomenon $(p=0.053)$ and delusions of theft $(p=0.176)$ were more common in PDD.

We analyzed cognitive performance in relation to behavioral symptoms of bvFTD and PDD patients. In the bvFTD group, FAB score was significantly lower in patients with neglect of hygiene $(p=0.04)$, loss of interest $(p=0.021)$, and loss of smell $(p=0.001)$, but higher in those who got upset if their routine was disrupted $(p=0.04)$ and who had verbal stereotypies/ perseverations $(p=0.015)$. Patients with hypersomnia had a higher BMSE score $(p=$ 0.026). Although not statistically significant, patients with hyposomnia had lower BMSE and FAB scores. In the PDD group (Table 4), FAB scores were significantly lower in patients with disinhibition $(p=0.019)$ and hyposomnia $(p=0.043)$, and higher in patients with excessive worrying $(p=0.02)$ and verbal stereotypies/perseverations $(p=0.007)$. BMSE score was higher in patients who needed to do things immediately $(p=0.011)$, did excessive clockwatching ( $p=0.019)$ and paid excessive attention to detail ( $p=0.028)$, but it was much lower in patients with a loss of basic emotion $(p=0.002)$.

\section{Discussion}

Executive dysfunction is common in bvFTD and subcortical dementias, due to disturbances of the dorsolateral prefrontal cortex and its subcortical connections. Patients with bvFTD often have associated parkinsonism and other motor features as well as incontinence, and patients with PDD exhibit behavioral changes like depression, apathy, anxiety, etc., sometimes making it difficult to make a diagnosis. In an attempt to find differentiating features between these 2 dementia syndromes, we explored the behavioral profiles of these patients. We recruited clinically diagnosed bvFTD patients regularly attending follow-up for $>1$ year at the Cognitive Clinic, and IPD patients regularly attending follow-up for $>5$ years at the MDC who had developed cognitive dysfunction that met the criteria for PDD. This process was to improve the diagnostic certainty of selected patients.

Although the bvFTD patients were younger than the PDD patients, they were cognitively more impaired. Clinical manifestation of bvFTD is dominated by altered behavior and changes in social cognition. In our study, several behavioral symptoms showed very high sensitivity for bvFTD. The most sensitive features favoring bvFTD were: loss of basic and social emotions, selfishness, disinhibition, neglect of personal hygiene, loss of awareness of pain, loss of discrimination of food, wandering, pacing, utilization phenomena, failure to recognize objects, and mutism. The presence of these features makes a diagnosis of bvFTD more likely. Neuropsychiatric symptoms like suspiciousness, the false belief that people were in their home, and visual illusions/ hallucinations were more often observed in PDD patients, with significant discriminating value. These features clearly differentiate bvFTD and PDD patients.

We found loss of basic emotion, selfishness, and social avoidance to be common in our bvFTD cohort and this helped in distinguishing bvFTD from PDD. Loss of embarrassment was also an important feature of social cognition that favored a diagnosis of bvFTD. Similar observations have been made by other researchers as well $[4,11,20]$. While IPD and PDD patients also exhibit features of apathy and a lack of empathy, these do not manifest often, appear late, and often mixed with depression [21-24]. Another prominent discriminatory symptom of impaired social cognition was disinhibition ( 95 vs. $35 \%, p<0.001$, OR 35.29). Disinhibition has also been described in other studies [11, 20,25]. Marked disinhibition is rare in PDD, and 
reported frequencies vary from 8 to $24 \%[24,26]$. The amygdala and orbitofrontal and medial prefrontal cortices (including the anterior cingulate cortex) are responsible for Theory of Mind (TofM). TofM is the ability of a person to understand what others are thinking and read their emotions. This is important for empathy, and a lack of it is an important feature of bvFTD. Neglect of personal hygiene was another discriminating feature observed in a relatively high proportion of the patients with bvFTD (85 vs. 30\%, $p=0.001$, OR 13.22). Other studies have also reported a high proportion (87-100\%) of bvFTD patients with symptom [4, $11]$, and it is rare in patients with PDD where it is often mixed with depression or apathy [22, 25-27].

Early loss of insight was an important feature of bvFTD and many researchers have reported this, in $25-100 \%$ of patients $[11,28]$; in PDD, however, it is relatively uncommon [22]. Loss of insight was observed in a large proportion of our bvFTD (95\%) and PDD (80\%) patients. That many of our PDD patients had loss of insight was possibly due to the manner the question was framed to elicit insightfulness. Rather than simply asking about their awareness of the disease, the question was whether the subject reacted to difficulties by becoming upset, distressed, or anxious, losing confidence, or withdrawing, and this might have elicited a more positive response from the PDD patients. Additionally, apathy (65\%) and hidden depression might also have contributed in these patients.

Exaggerated emotion, aggressiveness, and irritability were present in $25-70 \%$ of the bvFTD patients and 15-55\% of the PDD patients. A similar excess of emotional responses has also been reported in other studies [11, 22, 24, 28, 29].

Like other studies [30,31], altered sensory perception including the lack of pain sensation was found in a significantly high proportion of our bvFTD patients. Hyposmia was less frequent in the bvFTD (55\%) patients than in the PDD (60\%) patients. Hyposmia is a prominent nonmotor symptom, occurring in about $80-90 \%$ of PD patients [32, 33]. Although selfreporting, or reporting by the caregiver, may not provide a true account about smell perception, our observation of an abnormal perception of smell in a high proportion of the patients with bvFTD needs to be validated by proper evaluation in future studies.

Abnormal eating and vegetative behaviors were observed more frequently in bvFTD than PDD, similar to the reports of other investigators [11,13], although sweet-tongue and food faddism were not common in our bvFTD patients. A lack of taste discrimination, increased consumption of alcohol, and increased smoking clearly discriminated bvFTD from PDD. Altered eating behaviors are relatively uncommon in PDD. Several studies observed that PDD patients lose weight (20-30\%), and that this is related to changed eating habits, bradykinesia, an altered perception of taste and smell, or the effect of medication [13, 24, 34]. Recent changes in patterns of smoking or drinking, or starting these habits, are behavioral features strongly suggesting bvFTD rather than any subcortical dementia. Eating continuously when food is present, food-cramming, seeking out food, stealing food from others' plates, etc. were observed in a higher proportion of our bvFTD patients than our PDD patients, but did not have discriminatory value.

Wandering ( 70 vs. $20 \%, p=0.004$, OR 9.33) and pacing ( 50 vs. $10 \%, p=0.014$, OR 9) were reported more frequently in our bvFTD patients than in the PDD patients. These are also reported at similar frequencies in bvFTD by others $[11,31]$, but generally do not manifest in PDD. Although poorly understood, repetitive motor behavior is considered to be due to a disruption of coordinated function within the basal ganglia or corticostriatal structures [35].

Alteration of sexual behavior was also observed more commonly in our bvFTD patients, with $50 \%$ reported as having hyposexuality and $20 \%$ as having hypersexuality. A similar observation was made by Bathgate et al. [11] with hyposexuality in 58\% and hypersexuality in $19 \%$. One Indian study observed this behavior in $10 \%$ of their bvFTD patients [36]. Among our PDD patients, $30 \%$ reported hyposexuality and $10 \%$ reported hypersexuality. Hypersex-

\section{Karger'}


uality has been reported in around 3.5\% of IPD subjects as part of an impulse control disorder (ICD) [37]. Culturally, Indians are shy of expressing their sexual habits publicly. Moreover, hyposexuality is reported in many chronic diseases and may not be a feature specific to any disease. A change in sleep pattern was found in nearly equal frequency in both groups $(80 \%$ in bvFTD and $75 \%$ in PDD), with no discriminatory value. Similar to in other neurodegenerative diseases, hypersomnolence is reported in $30-47 \%$ of patients with bvFTD $[11,38]$. The literature suggests that $80 \%$ of IPD patients suffer from insomnia [39], while $50 \%$ experience excessive daytime sleepiness [40], and 30-90\% exhibit REM sleep behavior disorder [41].

Although repetitive and compulsive behaviors were more commonly found in our bvFTD cohort, we did not find them to discriminate bvFTD from PDD. Mendez and Perryman [28] observed perseverative and stereotyped behavior including compulsive-like acts in $45.3 \%$ of FTD patients at presentation, and this increased to $88.7 \%$ at the 2 -year follow-up. ICDs are observed in IPD patients receiving dopa-agonists and these include compulsive gambling, buying, sexual, and eating behaviors, punding (stereotyped, repetitive, purposeless behaviors), and hobbyism (e.g., compulsive internet use, artistic endeavors, and writing) [42]. ICDs result from a dysregulation of the mesocorticolimbic dopamine system and alterations in the opiate and serotonin systems [42]. Around $13.6 \%$ of IPD patients report ICDs while on dopa-agonists [43]. However, there are limited data about this characteristic behavior in both PDD and bvFTD patients. Chiu et al. [24] showed that 16\% of PDD patients had repetitive behaviors. Another compulsive behavior observed in IPD patients receiving short-acting potent dopaminergic drugs like L-dopa is dopamine dysregulation syndrome, which manifests as compulsive medication overuse [44].

Environmental dependency-related behaviors were observed in both groups. Among these, utilization behavior was significantly higher in bvFTD, observed in $35 \%$ of the patients, but not in any of the PDD patients ( $p=0.008$, OR 22.78), suggesting this to be a strong discriminating feature. This is a very interesting clinical sign observed in $>75 \%$ of bvFTD patients in an Indian study [45], but others have reported that this behavior occurs less frequently [5, $11,15,28]$.

The questionnaires also tried to assess cognition and spatial function; 70\% of the bvFTD patients and $60 \%$ of the PDD patients responded that they had problems with cognition and spatial dysfunction. What is intriguing is that many of our bvFTD caregivers responded positively to the questions relating to disturbance in visuospatial and perceptual functions. The symptoms related to visuospatial-perceptual function was detected in $65 \%$ of bvFTD and $40 \%$ of PDD patients. As this was a questionnaire-based assessment and our bvFTD cohort were more demented than the PDD patients, the result might not be a true indication of the deficits in this sphere of cognition and would require a more detailed evaluation. Interestingly, mutism was found to be significantly more common in bvFTD and is therefore a discriminatory feature.

Psychotic symptoms were more prominent in PDD (80\%) than in bvFTD (50\%). Delusional behaviors with suspiciousness, the belief that someone is in the home, and visual illusions/hallucinations were found to be significantly more frequent in PDD patients than in bvFTD patients, making these notable discriminatory features. Psychotic symptoms, particularly visual hallucinations, are very characteristic of PDD and dementia with Lewy bodies, primarily because of the impact on areas of visual association. Delusions and hallucinations have been reported in $\mathrm{PD}$, in $30-40 \%$ and $45-65 \%$ of patients, respectively [22, 24]. These studies also suggest that visual hallucinations in PD predict the development of dementia. Delusions and hallucinations are relatively uncommon in bvFTD, except in some patients such as those with bvFTD due to the C9orf72 mutation [46].

While correlating cognitive performance with behavioral symptoms, we observed a trend. In the PDD group, although not statistically significant, patients with some of the behav-

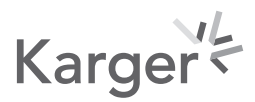


ioral symptoms tended to have a lower mean FAB score ( $<8$; Table 4$)$. These symptoms were: loss of basic emotion, loss of embarrassment, social avoidance, neglect of hygiene, and loss of interest, which might be considered as "negative" symptoms. On the other hand, patients with some of the other symptoms had higher mean FAB scores (>8). These symptoms were: verbal stereotypies/perseverations, excessive worrying, the need to do things immediately, exaggerated emotional display, irritability, aggression, and seeking out social contact, which might be considered as "positive" symptoms. This indicates that, in PDD, more advanced disease with lower FAB scores might placate the expressive or "positive" behavioral symptoms to some extent. However, it was not possible to generalize, as the FAB scores were significantly lower in the patients displaying disinhibition. Moreover, it was difficult to comment on the bvFTD patients, as most of them had very low FAB scores. Hence, these findings should be evaluated in larger studies to arrive at an accurate conclusion.

Dementia with a predominantly frontal dysexecutive pattern may be seen in both cortical (e.g., bvFTD) and subcortical (e.g., PDD) disorders. Behavioral symptoms form an important part of such dementias, and our study suggests that some of these behaviors have a discriminatory value for differentiating between these disorders. We found that several behavioral symptoms increased the odds for bvFTD versus PDD. In a previous study, Moretti et al. [47] observed prominent abnormalities in personality and social conduct with a significant loss of insight in frontal-lobe dementia when compared to subcortical vascular dementia. Hence, although larger-scale comparative studies are required, it can be inferred that behavioral symptoms help to distinguish cortical dementia with frontal-lobe dysfunction from subcortical dementia.

This study has a few limitations. The small sample size was a major limitation which could have impacted the power of the study. As features of degenerative disease evolve over time, a cross-sectional assessment does not exactly depict the full spectrum of behavior of these patients. Moreover, the lack of pathological confirmation of patients was another limitation. However, we carefully chose our patients after a long follow-up before recruiting them, so as to ensure a better diagnosis. The systematic analysis of symptoms of behavioral changes was the strength of the study.

To conclude, we observed significant differences in behavioral symptoms between bvFTD and PDD patients, with several symptoms showing higher frequency in bvFTD. It may be that the degeneration of the prefrontal cortex is mostly responsible for frontal behavioral symptoms, and that these are relatively uncommon in subcortical diseases despite having rich to-and-fro connections between the two.

\section{Acknowledgements}

We acknowledge Prof. Avijit Hazra for his help in carrying out the statistical analysis.

\section{Statement of Ethics}

This research work was conducted ethically in accordance with the World Medical Association Declaration of Helsinki, after getting clearance from the Institutional Ethics Committee of the IPGME\&R, Kolkata, India (No. Inst/IEC/2015/287 dated April 6, 2015). Written informed consent was obtained from patients and their caregivers or legally acceptable representatives before recruiting them for the study.

\section{Karger's}




\section{Conflict of Interest Statement}

Authors declare there were no conflicts of interest.

\section{Author Contributions}

All listed authors contributed intellectually for the study and manuscript preparation. D.S.: data collection, tabulation, and statistical analysis; A.M.: statistical analysis, and manuscript preparation; A.R.: monitoring and supervision of data collection, and manuscript preparation; A.B.: conceptualization of study and methodology, supervision of data collection, and drafting of manuscript.

\section{References}

1 Cummings JL. Frontal-subcortical circuits and human behavior. Arch Neurol. 1993 Aug;50(8):873-80.

2 Paulsen JS, Stout JC, DeLaPena J, Romero R, Tawfik-Reedy Z, Swenson MR, et al. Frontal behavioral syndromes in cortical and subcortical dementia. Assessment. 1996;3(3):327-37.

3 Alexander GE, DeLong MR, Strick PL. Parallel organization of functionally segregated circuits linking basal ganglia and cortex. Annu Rev Neurosci. 1986;9(1):357-81.

4 Levy JA, Chelune GJ. Cognitive-behavioral profiles of neurodegenerative dementias: beyond Alzheimer's disease. J Geriatr Psychiatry Neurol. 2007 Dec;20(4):227-38.

5 Caballol N, Martí MJ, Tolosa E. Cognitive dysfunction and dementia in Parkinson disease. Mov Disord. 2007 Sep;22(Suppl 17):S358-66.

6 Rascovsky K, Hodges JR, Knopman D, Mendez MF, Kramer JH, Neuhaus J, et al. Sensitivity of revised diagnostic criteria for the behavioural variant of frontotemporal dementia. Brain. 2011 Sep;134(Pt 9):2456-77.

7 Pąchalska M, Bidzan L, Łukowicz M, Bidzan M, Markiewicz K, Jastrzębowska G, et al. Differential diagnosis of behavioral variant of fronto-temporal dementia (bvFTD). Med Sci Monit. 2011 Jun;17(6):CR311-21.

8 Pinto C, Seethalakshmi R. Behavioral and psychological symptoms of dementia in an Indian population: comparison between Alzheimer's disease and vascular dementia. Int Psychogeriatr. 2006 Mar;18(1):87-93.

9 Kazui H, Yoshiyama K, Kanemoto H, Suzuki Y, Sato S, Hashimoto M, et al. Differences of behavioral and psychologicalsymptoms of dementia in disease severity in four major dementias. PLoS One.2016 Aug;11(8):e0161092.

10 Mukherjee A, Biswas A, Roy A, Biswas S, Gangopadhyay G, Das SK. Behavioural and psychological symptoms of dementia: correlates and impact on caregiver Distress. Dement Geriatr Cogn Disord Extra. 2017 Nov; $7(3)$ : 354-65.

11 Bathgate D, Snowden JS, Varma A, Blackshaw A, Neary D. Behaviour in frontotemporal dementia, Alzheimer's disease and vascular dementia. Acta Neurol Scand. 2001 Jun;103(6):367-78.

12 Bozeat S, Gregory CA, Ralph MA, Hodges JR. Which neuropsychiatric and behavioural features distinguish frontal and temporal variants of frontotemporal dementia from Alzheimer's disease? J Neurol Neurosurg Psychiatry. 2000 Aug;69(2):178-86.

13 Ikeda M, Brown J, Holland AJ, Fukuhara R, Hodges JR. Changes in appetite, food preference, and eating habits in frontotemporal dementia and Alzheimer's disease. J Neurol Neurosurg Psychiatry. 2002;73:371-6.

14 Emre M, Aarsland D, Brown R, Burn DJ, Duyckaerts C, Mizuno Y, et al. Clinical diagnostic criteria for dementia associated with Parkinson's disease. Mov Disord. 2007 Sep;22(12):1689-707.

15 Das SK, Banerjee TK, Mukherjee CS, Bose P, Biswas A, Hazra A, et al. An urban community-based study of cognitive function among non-demented elderly population in India. Neurol Asia. 2006;11:37-48.

16 Keshree NK, Kumar S, Basu S, Chakrabarty M, Kishore T. Adaptation of the Western Aphasia Battery in Bangla. Psychol Lang Commun. 2013;17(2):189-201.

17 Dubois B, Slachevsky A, Litvan I, Pillon B. The FAB: a Frontal Assessment Battery at bedside. Neurology. 2000 Dec;55(11):1621-6.

18 Bandyopadhyay TK, Biswas A, Roy A, Guin DS, Gangopadhyay G, Sarkhel S, et al. Neuropsychiatric profiles in patients with Alzheimer's disease and vascular dementia. Ann Indian Acad Neurol. 2014 Jul;17(3):325-30.

19 Pal A, Biswas A, Pandit A, Roy A, Guin D, Gangopadhyay G, et al. Study of visuospatial skill in patients with dementia. Ann Indian Acad Neurol. 2016 Jan-Mar;19(1):83-8.

20 Le Ber I, Guedj E, Gabelle A, Verpillat P, Volteau M, Thomas-Anterion C, et al.; French research network on FTD/ FTD-MND. Demographic, neurological and behavioural characteristics and brain perfusion SPECT in frontal variant of frontotemporal dementia. Brain. 2006 Nov;129(Pt 11):3051-65.

21 Starkstein SE, Mayberg HS, Preziosi TJ, Andrezejewski P, Leiguarda R, Robinson RG. Reliability, validity, and clinical correlates of apathy in Parkinson's disease. J Neuropsychiatry Clin Neurosci. 1992;4(2):134-9. 
22 Aarsland D, Andersen K, Larsen JP, Lolk A, Kragh-Sørensen P. Prevalence and characteristics of dementia in Parkinson disease: an 8-year prospective study. Arch Neurol. 2003 Mar;60(3):387-92.

23 Levy ML, Cummings JL, Fairbanks LA, Masterman D, Miller BL, Craig AH, et al. Apathy is not depression. J Neuropsychiatry Clin Neurosci. 1998;10(3):314-9.

24 Chiu PY, Tsai CT, Chen PK, Chen WJ, Lai TJ. Neuropsychiatric symptoms in Parkinson's disease dementia are more similar to Alzheimer's disease than dementia with Lewy Bodies: a case-control study. PLoS One. 2016 Apr;11(4):e0153989.

25 Tan YL, Ng A, Kandiah N. Frontotemporal dementia in southeast Asia: a comparative study. Dement Geriatr Cogn Disord Extra. 2013 Jan;3(1):1-9.

26 Oh YS, Lee JE, Lee PH, Kim JS. Neuropsychiatric symptoms in Parkinson's disease dementia are associated with increased caregiver burden. J Mov Disord. 2015 Jan;8(1):26-32.

27 Guo X, Song W, Chen K, Chen X, Zheng Z, Cao B, et al. Associations between neuropsychiatric symptoms and cognition in Chinese idiopathic Parkinson's disease patients. J Clin Neurosci. 2015 Mar;22(3):578-82.

28 Mendez MF, Perryman KM. Neuropsychiatric features of frontotemporal dementia: evaluation of consensus criteria and review. J Neuropsychiatry Clin Neurosci. 2002;14(4):424-9.

29 Siddiqui MS, Fernandez HH, Garvan CW, Kirsch-Darrow L, Bowers D, Rodriguez RL, et al. Inappropriate crying and laughing in Parkinson disease and movement disorders. World J Biol Psychiatry. 2009;10(3):234-40.

30 Cohen OS, Vakil E, Tanne D, Molshatzki N, Nitsan Z, Hassin-Baer S. The frontal assessment battery as a tool for evaluation of frontal lobe dysfunction in patients with Parkinson disease. J Geriatr Psychiatry Neurol. 2012 Jun;25(2):71-7.

31 Snowden JS, Bathgate D, Varma A, Blackshaw A, Gibbons ZC, Neary D. Distinct behavioural profiles in frontotemporal dementia and semantic dementia. J Neurol Neurosurg Psychiatry. 2001 Mar;70(3):323-32.

32 Chaudhuri KR, Prieto-Jurcynska C, Naidu Y, Mitra T, Frades-Payo B, Tluk S, et al. The nondeclaration of nonmotor symptoms of Parkinson's disease to health care professionals: an international study using the nonmotor symptoms questionnaire. Mov Disord. 2010 Apr;25(6):704-9.

33 Tinazzi M, Del Vesco C, Fincati E, Ottaviani S, Smania N, Moretto G, et al. Pain and motor complications in Parkinson's disease. J Neurol Neurosurg Psychiatry. 2006 Jul;77(7):822-5.

34 Munhoz RP, Moro A, Silveira-Moriyama L, Teive HA. Non-motor signs in Parkinson's disease: a review. Arq Neuropsiquiatr. 2015 May;73(5):454-62.

35 Cipriani G, Vedovello M, Ulivi M, Nuti A, Lucetti C. Repetitive and stereotypic phenomena and dementia. Am J Alzheimers Dis Other Demen. 2013 May;28(3):223-7.

36 Ghosh A, Dutt A, Ghosh M, Bhargava P, Rao S. Using the revised diagnostic criteria for frontotemporal dementia in India: evidence of an advanced and florid disease. PLoS One. 2013 Apr;8(4):e60999.

37 Codling D, Shaw P, David AS. Hypersexuality in Parkinson's disease: systemic review and repot of 7 new cases. Mov Disord Clin Pract (Hoboken). 2015 Apr;2(2):116-26.

38 Anderson KN, Hatfield C, Kipps C, Hastings M, Hodges JR. Disrupted sleep and circadian patterns in frontotemporal dementia. Eur J Neurol. 2009 Mar;16(3):317-23.

39 Lees AJ, Blackburn NA, Campbell VL. The night time problems of Parkinson's disease. Clin Neuropharmacol. 1998;1:512-9.

40 De Cock VC, Vidailhet M, Arnulf I. Sleep disturbances in patients with parkinsonism. Nat Clin Pract Neurol. 2008 May; 4(5):254-66.

41 Boeve BF, Silber MH, Ferman TJ, Lucas JA, Parisi JE. Association of REM sleep behavior disorder and neurodegenerative disease may reflect an underlying synucleinopathy. Mov Disord. 2001 Jul;16(4):622-30.

42 Evans AH, Lees AJ. Dopamine dysregulation syndrome in Parkinson's disease. Curr Opin Neurol. 2004 Aug; $17(4): 393-8$.

43 Maia AF, Pinto AS, Barbosa ER, Menezes PR, Miguel EC. Obsessive-compulsive symptoms, obsessive-compulsive disorder, and related disorders in Parkinson's disease. J Neuropsychiatry Clin Neurosci. 2003;15(3):371-4.

44 Weintraub D, Claassen DO. Impulse Control and Related Disorders in Parkinson's Disease. Int Rev Neurobiol. 2017;133:679-717.

45 Ghosh A, Dutt A, Bhargava P, Snowden J. Environmental dependency behaviours in frontotemporal dementia: have we been underrating them? J Neurol. 2013 Mar;260(3):861-8.

46 Devenney EM, Ahmed RM, Halliday G, Piguet 0, Kiernan MC, Hodges JR. Psychiatric disorders in C9orf72 kindreds: study of 1,414 family members. Neurology. 2018 Oct;91(16):e1498-507.

47 Moretti R, Torre P, Antonello RM, Cattaruzza T, Cazzato G, Bava A. Frontal lobe dementia and subcortical vascular dementia: a neuropsychological comparison. Psychol Rep. 2005 Feb; 96(1):141-51. 